EPJ Web of Conferences 13, 07001 (2011)

DOI: $10.1051 /$ epjconf/20111307001

(c) Owned by the authors, published by EDP Sciences, 2011

\title{
Collective Flow and Mach Cones with transport
}

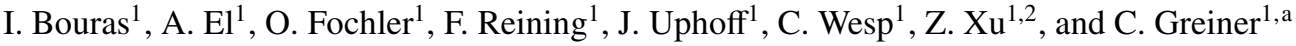 \\ 1 Institut fuer Theoretische Physik, Goethe Universitaet Frankfurt, \\ Max-von-Laue-Strasse.1, D-60438 Frankfurt am Main, Germany \\ 2 Frankfurt Institute for Advanced Studies, \\ Ruth-Moufang-Strasse 1, D-60438 Frankfurt am Main, Germany
}

\begin{abstract}
Fast thermalization and a strong build up of elliptic flow of QCD matter were investigated within the pQCD based $3+1$ dimensional parton transport model BAMPS including bremsstrahlung $2 \leftrightarrow 3$ processes. Within the same framework quenching of gluonic jets in Au+Au collisions at RHIC can be understood. The development of conical structure by gluonic jets is investigated in a static box for the regimes of small and large dissipation. Furthermore we demonstrate two different approaches to extract the shear viscosity coefficient $\eta$ from a microscopical picture.
\end{abstract}

\section{Introduction}

The large value of the elliptic flow $v_{2}$ measured in experiments at the Relativisitc Heavy Ion collider (RHIC)[1-3] suggests, that in the hot and dense fireball equilibration of quarks and gluons occurs on a very short time scale $\leq 1$ $\mathrm{fm} / \mathrm{c}$. This results into the assumption, that this locally thermalized matter, the quark gluon plasma (QGP), behaves like a nearly perfect fluid. In this case quarks and gluons should be rather strongly coupled and the QGP should have a very small viscosity to entropy ratio $\eta / s$. This value might in fact be close to the conjectured lower bound $\eta / s=$ $1 /(4 \pi)$, obtained from a correspondence between conformal field theory (CFT) and string theory in an Anti-deSitter space (AdS) [4].

The phenomenon of jet-quenching was another important discovery at RHIC [5]. Hadrons with high transverse momenta are suppressed in $A u+A u$ collisions with respect to a scaled $p+p$ reference $[6,7]$. This quenching of jets is commonly attributed to energy loss on the partonic level as the hard partons produced in initial interactions are bound to traverse the QGP created in the early stages of heavy-ion collisions (HIC). In addition, very exciting jet-associated particle correlations were observed [8], which might be the result of a conical emission off propagating shock waves in form of Mach Cones. These Mach Cones might be induced by high-energetic partons traversing the expanding medium [9].

A large class of phenomena in heavy-ion collisions can be investigated within the framework of the kinetic transport theory. Among others, the relativistic pQCD-based on-shell parton transport model BAMPS (Boltzmann Approach to Multiparton Scatterings) [10] was developed to describe the early QGP stage of a heavy-ion collision (HIC). Using BAMPS early thermalization of gluons within $\tau<1$

\footnotetext{
${ }^{a}$ e-mail: carsten.greiner@th.physik.uni-frankfurt.de
}

$\mathrm{fm} / \mathrm{c}$ was demonstrated in $\mathrm{Au}+\mathrm{Au}$ collisions at $\sqrt{s_{N N}}=$ $200 \mathrm{GeV}$ employing Glauber initial conditions of minijets and the coupling constant $\alpha_{s}=0.3-0.6$. In addition to the elastic pQCD $g g \leftrightarrow g g$ processes, pQCD-based bremsstrahlung $g g \leftrightarrow g g g$ was included. These were shown to be essential for the achievement of local thermal equilibrium at that short time scale. The fast thermalization happens also in a similar way with Color Glass Condensate initial conditions [11].

BAMPS has been recently applied to calculate elliptic flow and jet quenching at RHIC energies [12] for the first time in a consistent and fully pQCD-based microscopic transport model. Both key observables could be addressed on the partonic level within a common setup. The left panel of Fig. 1 shows that the medium simulated in the parton cascade BAMPS exhibits a sizable degree of elliptic flow in agreement with experimental findings at RHIC as discussed in Ref. [13,14].

Extraction of the shear viscosity over entropy density ratio $\eta / s$ from BAMPS simulations confirmed the essential importance of inelastic processes. Bremsstrahlung and back reaction processes lower the shear viscosity to entropy density ratio significantly, by a factor of 7 , compared to the values obtained if only elastic collisions are considered $[15,16]$. For $\alpha_{s}=0.3$ one finds $\eta / s=0.13$, whereas for $\alpha_{S}=0.6$ the obtained value matches the lower bound of $\eta / s=1 /(4 \pi)$ from the AdS/CFT conjecture.

In these proceedings we show some recent descriptions and developments of different phenomena in relativistic HIC using BAMPS. Due to the large momentum scales involved, the energy loss of partonic jets can be treated in terms of perturbative QCD (pQCD) and most theoretical schemes attribute the main contribution to partonic energy loss to radiative processes [17]. In addition, the possible propagation of Mach Cones in the QGP induced by such high-energetic partons can be studied. Considering the ear- 
lier works, in which the effects of dissipation on relativistic shock waves were investigated [18-20], we demonstrate the transition of Mach Cones from ideal to the viscous ones. It is a major challenge to combine jet physics on the one hand and bulk evolution on the other hand within a common framework. In the end we demonstrate two new independent methods to extract numerically the shear viscosity to entropy density ratio $\eta / s$. One of them is based on the classical picture of a linear velocity gradient, where the shear viscosity coefficient $\eta$ can be calculated from the local deformation of the distribution function. The other method is based on the Green - Kubo relation, where shear viscosity is obtained from the autocorrelation function of equilibrium fluctuations.

\section{Jet Quenching in Au+Au collisions at 200 AGeV}

For simulations of Jet Quenching in heavy ion collisions the initial gluon distributions are sampled according to a mini-jet model with a lower momentum cut-off $p_{0}=1.4$ $\mathrm{GeV}$ and a $K$-factor of 2 . The test particle method [10] is employed to ensure sufficient statistics. Quarks are discarded after sampling the initial parton distribution since currently a purely gluonic medium is considered. To model the freeze out of the simulated fireball, free streaming is applied to regions where the local energy density has dropped below a critical energy density $\varepsilon_{c}\left(\varepsilon_{c}=1.0 \mathrm{GeV} / \mathrm{fm}^{3}\right.$ unless otherwise noted).

The right panel of Fig. 1 shows the gluonic $R_{A A}$ simulated in BAMPS for central, $b=0 \mathrm{fm}$, collisions. It is roughly constant at $R_{A A}^{\text {gluons }} \approx 0.053$ and in reasonable agreement with analytic results for the gluonic contribution to the nuclear modification factor $R_{A A}$ [17], though the suppression of gluon jets in BAMPS appears to be slightly stronger. We expect improved agreement in future studies when employing a carefully averaged $\langle b\rangle$ that will be better suited for comparison to experimental data than the strict $b=0 \mathrm{fm}$ case.

We have computed the gluonic $R_{A A}$ for non-central Au + Au collisions at the RHIC energy of $\sqrt{s}=200 \mathrm{AGeV}$ with a fixed impact parameter $b=7 \mathrm{fm}$ (Fig. 2), which roughly corresponds to $(20-30) \%$ experimental centrality. A comparison in terms of the magnitude of the jet suppression for $b=7 \mathrm{fm}$ is difficult since there are no published analytic results available to compare to. Taking the ratio of the $b=7 \mathrm{fm}$ to the $b=0 \mathrm{fm}$ results as a rough guess indicates that the decrease in quenching is more pronounced in BAMPS compared to experimental data. The ratio of the nuclear modification factor between central $(0-10) \%$ and more peripheral $(20-30) \%$ collisions is $\left.R_{A A}\right|_{0 \%-10 \%} /\left.R_{A A}\right|_{20 \%-30 \%} \approx 0.6$ for the experimental data, while for the BAMPS results $\left.R_{A A}\right|_{b=0 \mathrm{fm}} /\left.R_{A A}\right|_{b=7 \mathrm{fm}} \approx$ 0.4 . However, the issue of detailed quantitative comparison needs to be re-investigated once light quarks and a fragmentation scheme are included into the simulations.

To complement the investigations of $R_{A A}$ at a non-zero impact parameter $b=7 \mathrm{GeV}$, we have computed the el- liptic flow parameter $v_{2}$ for gluons at the same impact parameter and extended the range in transverse momentum up to roughly $p_{T} \approx 10 \mathrm{GeV}$, see left panel of Fig. 2. For this a critical energy density $\varepsilon_{c}=0.6 \mathrm{GeV} / \mathrm{fm}^{3}$ has been used, in order to be comparable to previous calculations of the elliptic flow within BAMPS. The $v_{2}$ of high $-p_{T}$ gluons is at first rising with $p_{T}$, but from $p_{T} \approx 4$ to $5 \mathrm{GeV}$ on, it begins to slightly decreases again. This behavior is in good qualitative agreement with recent RHIC data [23] that for charged hadrons shows $v_{2}$ to be rising up to $v_{2} \approx 0.15$ at $p_{T} \approx 3 \mathrm{GeV}$ followed by a slight decrease.

\section{Transition from ideal to dissipative Mach Cones}

In the early 1970s shock waves were theoretically predicted to occur in relativistic heavy-ion collisions (HIC) [24] and they have been experimentally investigated. There is an important issue in recent studies whether the small but finite viscosity allows the development of relativistic shocks in form of Mach Cones in such a hot and dense matter like the QGP. Within the framework of BAMPS studies were finished to answer the question whether shocks can develop with finite viscosity and how this will alter such a picture [18]. Within the relativistic Riemann problem it was shown that one dimensional shocks smears out if viscosity is large $[19,20]$. However, the expected viscosity in HIC seems to be small enough to allow a significant contribution of shocks in form of Mach Cones. In the following we report a very recent study.

Mach Cones, which are special phenomena of shock waves, have their origin in ideal hydrodynamics. A very weak perturbation in a perfect fluid induces sound waves which propagate with the speed of sound $c_{s}=\sqrt{d p / d e}$, where $p$ is the pressure and $e$ is the energy density. In the case where the perturbation with velocity $v_{\text {jet }}$ propagates faster than the generated sound waves, the sound waves lie on a cone. Considering a gas of massless particles, where $e=3 p$ and $c_{s}=1 / \sqrt{3}$, then the emission angle of the Mach Cone is given by $\alpha_{w}=\arccos \left(c_{s} / v_{\text {jet }}\right)=54,73^{\circ}$.

A stronger perturbation induces the propagation of the shock waves exceeding the speed of sound, therefore the emission angle changes and can be approximated by $\alpha \approx \arccos \left(v_{\text {shock }} / v_{\text {jet }}\right)$. Here

$$
v_{\text {shock }}=\left[\frac{\left(p_{1}-p_{0}\right)\left(e_{0}+p_{1}\right)}{\left(e_{1}-e_{0}\right)\left(e_{1}+p_{0}\right)}\right]^{\frac{1}{2}}
$$

is the velocity of the shock front, $p_{0}\left(e_{0}\right)$ the pressure (energy density) in the shock front region and $p_{1}\left(e_{1}\right)$ in the stationary medium itself. The expression has the following limits: For $p_{0}>>p_{1}$ we obtain $v_{\text {shock }} \approx 1$, whereas for a small perturbation, $p_{0} \approx p_{1}$, we get $v_{\text {shock }} \approx c_{s}$.

We employ the microscopic transport model BAMPS to investigate Mach Cones with different strength of dissipations in the medium using a jet moving in positive $z$ direction, initialized at $t=0 \mathrm{fm} / \mathrm{c}$ at the position $z=-0.8$ $\mathrm{fm}$. The jet is treated as a massless particle with zero spatial 

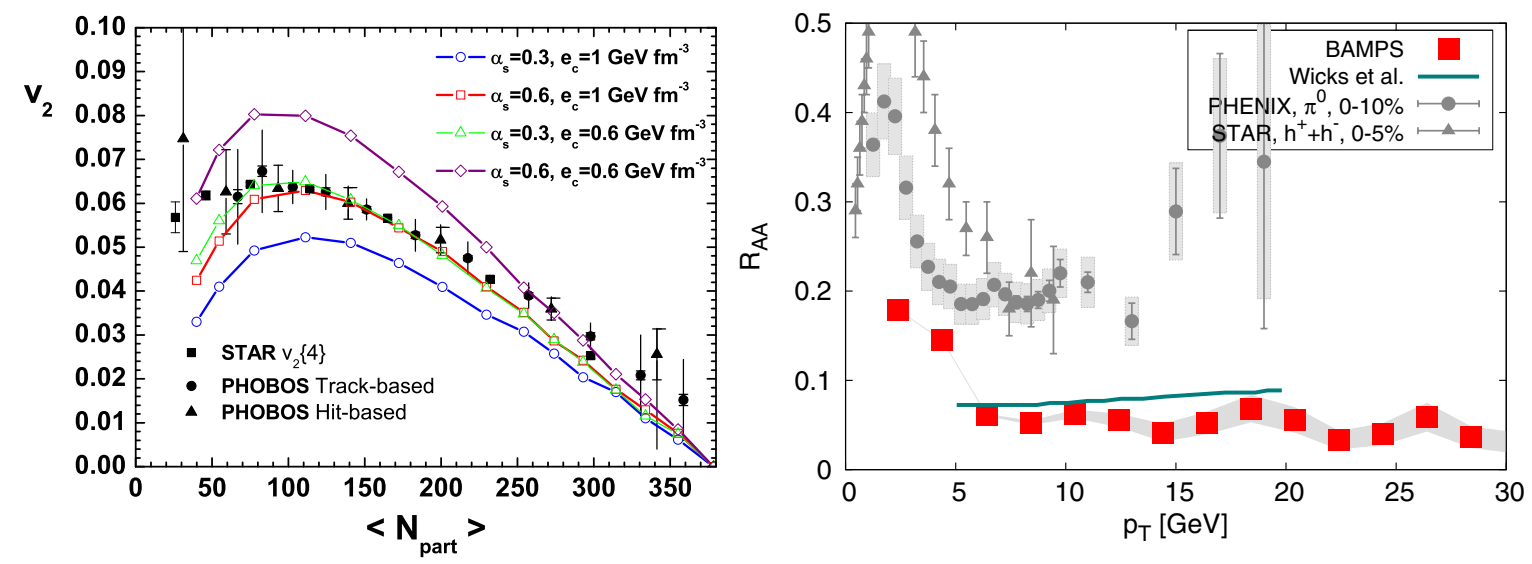

Fig. 1. Left panel: Elliptic flow $v_{2}$ as a function of the number of participants for $\mathrm{Au}+\mathrm{Au}$ at $200 \mathrm{AGeV}$ for different combinations of the strong coupling $\alpha_{s}$ and the critical energy density $\varepsilon_{c}$. See [14] for more information. Right panel: Gluonic $R_{A A}$ at midrapidity $(y \in[-0.5,0.5])$ as extracted from simulations for central Au+Au collisions at $200 \mathrm{AGeV}$. For comparison the result from Wicks et al. [17] for the gluonic contribution to $R_{A A}$ and experimental results from PHENIX [21] for $\pi^{0}$ and STAR [5] for charged hadrons are shown.
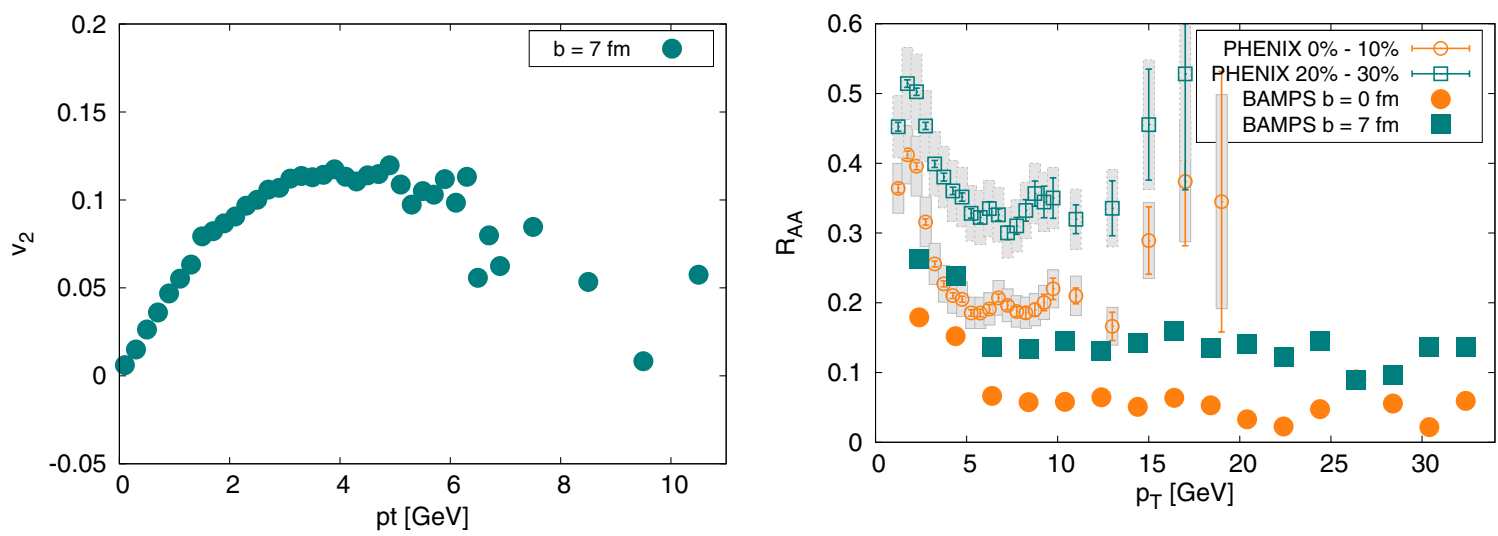

Fig. 2. Left panel: Elliptic flow $v_{2}$ for gluons in simulated Au+Au collisions at $200 \mathrm{AGeV}$ with $b=7 \mathrm{fm}$. $\varepsilon_{c}=0.6 \mathrm{GeV} / \mathrm{fm}^{3}$. See [22] fore information. Right panel: Gluonic $R_{A A}$ as extracted from BAMPS simulations for $b=0 \mathrm{fm}$ and $b=7 \mathrm{fm}, \varepsilon_{c}=1.0 \mathrm{GeV} / \mathrm{fm}{ }^{3}$. For comparison experimental results from PHENIX [21] for $\pi^{0}$ are shown for central $(0-10) \%$ and off-central $(20-30) \%$ collisions.

volume and zero transverse momentum, that is, $p_{z}=E_{\text {jet }}=$ $200 \mathrm{GeV}$ and $v_{\text {jet }}=1$. The energy and momentum deposition to the medium is realized via collisions with medium particles. In this scenario we neglect the deflection of the jet and it can not be stopped by the medium; its energy and momentum is set to its initial value after every collision.

All simulations are realized within a static and uniform medium of massless Boltzmann particles and $T=400$ $\mathrm{MeV}$. For this study we consider only binary scattering processes with an isotropic cross section among the bulk particles. To save computational runtime we reduce our problem to two dimensions. Here we choose the $x z$-plane and apply a periodic boundary condition in $y$-direction.

In Fig. 3 we demonstrate the transition from ideal Mach Cone to a highly viscous one by adjusting the shear viscosity over entropy density ratio in the medium from $\eta / s=$ $1 / 64 \pi \approx 0.005$ to $1 / \pi \approx 0.32$. The energy deposition of the jet is approximately $d E / d x=11-14 \mathrm{GeV} / \mathrm{fm}$. We show a snapshot at $t=2.5 \mathrm{fm} / \mathrm{c}$.

Using a small viscosity of $\eta / s=1 / 64 \pi$, we observe a strong collective behavior in form of a Mach Cone, as shown in the left panel of Fig.3. Due to the fact that the energy deposition is strong, the shock propagates faster than the speed of sound through the medium. For comparison, the ideal Mach Cone caused by a very weak perturbation is given by a solid line with its emission angle $\alpha_{w}=54,73^{\circ}$. Furthermore, a strong diffusion wake in direction of the jet, characterized by decreased energy density, and a head shock in the front are clearly visible.

If we increase the viscosity of the medium to larger values, shown in the middle and left panel of Fig.3, the typical Mach Cone structure smears out and vanishes completely. Due to stronger dissipation, the collective behavior gets weaker because of less particle interactions in the medium with a larger $\eta / s$. The results agree qualitatively with earlier studies $[18,20]$, where a smearing-out of the shock profile is observed with higher viscosity.

In addition to the scenario of a punch through jet we demonstrate in Fig.4 the scenario of a massless jet with finite energy which can also be deflected. Its initial energy is set to $p_{z}=E_{\text {jet }}=20 \mathrm{GeV}$, where the starting point is $z=-0.3 \mathrm{fm}$. We show the results for different viscosities, 


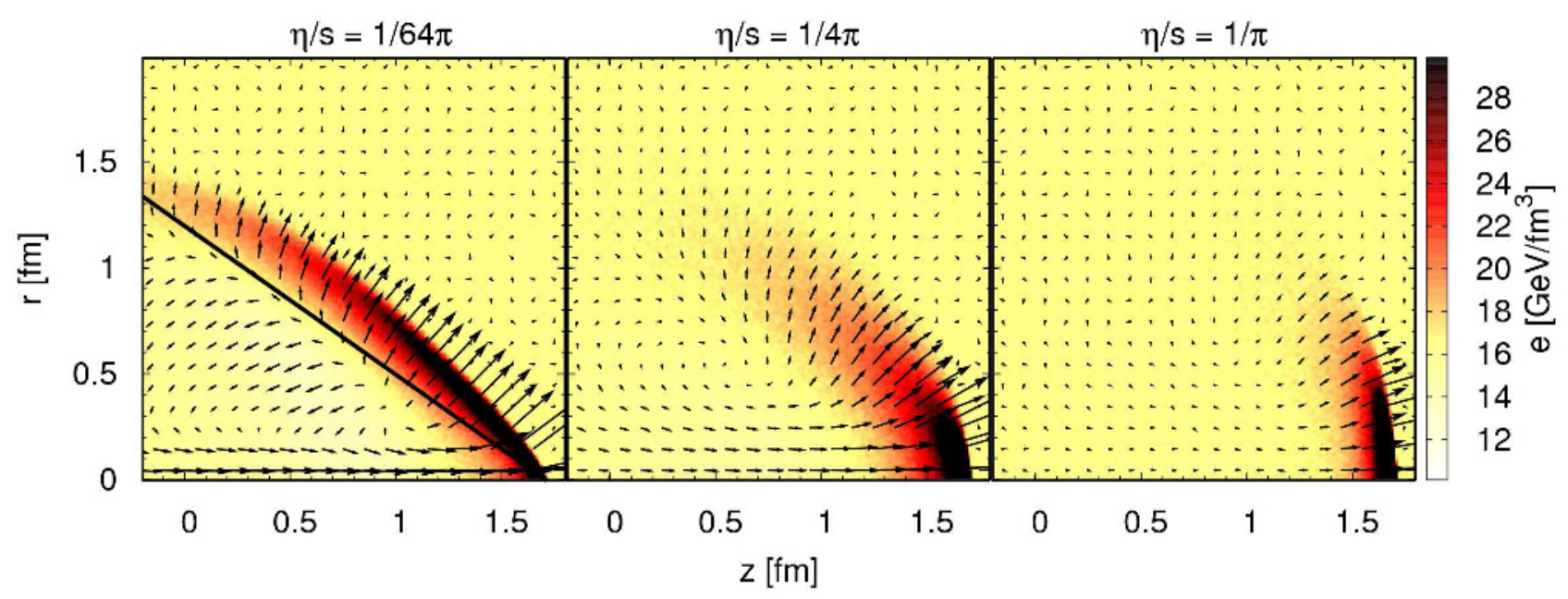

Fig. 3. (Color online) Scenario of a massless jet with $p_{z}=E_{\text {jet }}=200 \mathrm{GeV}$ in the punch through scenario - the shape of a Mach Cone shown for different viscosities of the medium, $\eta / s=1 / 64 \pi$ (left), $\eta / s=1 / 4 \pi$ (middle), $\eta / s=1 / \pi$ (right). The energy deposition of the jet is approximately $d E / d x=11-14 \mathrm{GeV} / \mathrm{fm}$. We show the energy density plotted together with the velocity profile. Additionally, in the left panel the linear ideal Mach Cone for a very weak perturbation is shown by a solid line; its emission angle is $\alpha_{w}=54,73^{\circ}$.

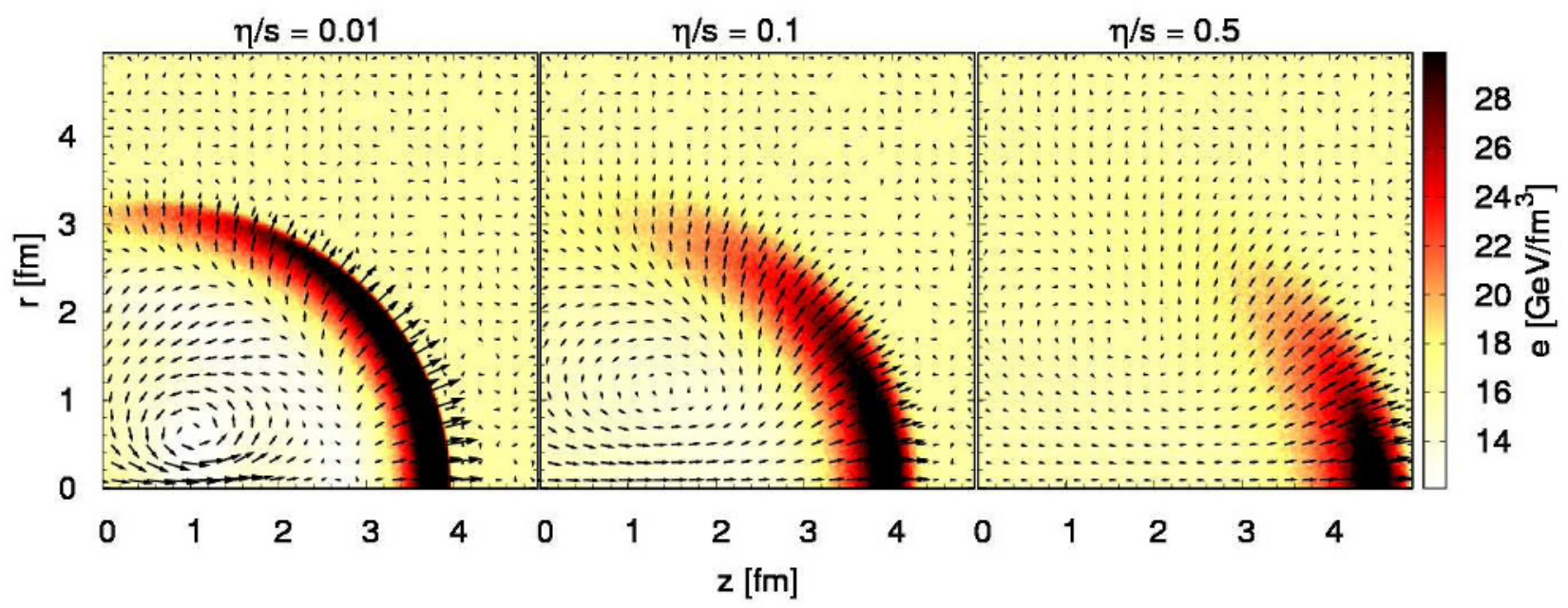

Fig. 4. (Color online) Scenarion of a deflectable jet with finite energy $p_{z}=E_{\text {jet }}=20 \mathrm{GeV}$ - Induced Mach Cone structure for different viscosities of the medium, $\eta / s=0.01$ (left), $\eta / s=0.1$ (middle), $\eta / s=0.5$ (right). We show the energy density plotted together with the velocity profile.

$\eta / s \approx 0.01$ to 0.5 at $t=5.0 \mathrm{fm} / \mathrm{c}$. In analogy to the results above we observe a clear Mach Cone structure for small viscosities and a smearing out with larger values of $\eta / s$. Only in the ideal case a strongly curved structure in which the building up of a strong vortex is visible. The physical meaning of these phenomena and also jets with the full pQCD cascade have to be explored in future studies [25].

\section{Extraction of shear viscosity from microscopic theory}

Whereas in viscous hydrodynamics the shear viscosity to entropy density ratio $\eta / s$ is the important input parameter, in microscopic transport theory the cross section or collision rate governs the behavior of the system. A big chal- lenge is to connect these both parameters e.g. by extracting the shear viscosity $\eta$ from transport calculations. There is a big interest to calculate the shear viscosity in a full pQCD simulation including inelastic processes, which was done in previous calculations $[15,16]$. In this section we present another two different methods to extract the shear viscosity $\eta$ numerically from transport calculations employing the transport model BAMPS.

The first method is motivated by the classical picture of a stationary velocity gradient. We introduce a particle system embedded between two plates moving in opposite $z$-direction represented by thermal reservoirs of particles with $\pm v_{\text {wall }}$. In $x$-direction the system has a size of $L$, in $y$ and $z$ direction the system is homogeneous. We assume the mean free path of the particles to be small compared to 

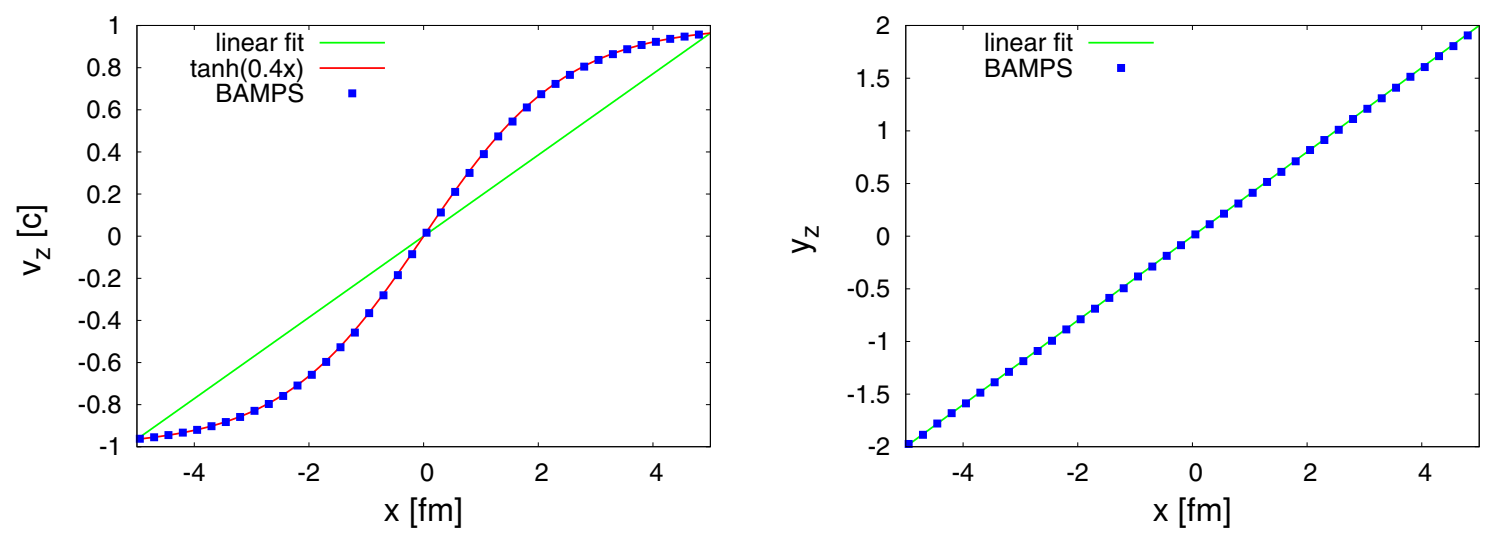

Fig. 5. On the left panel we show the velocity profile $v_{z}(x)$ extracted from BAMPS compared to a linear fit and a hyperbolic tangent, on the right panel we show the according rapidity profile $y_{\mathrm{z}}(x)$. We use $T=0.4 \mathrm{GeV}, L=10 \mathrm{fm}, \lambda_{\mathrm{mfp}}=0.01$ and $v_{\text {wall }}=0.964$.
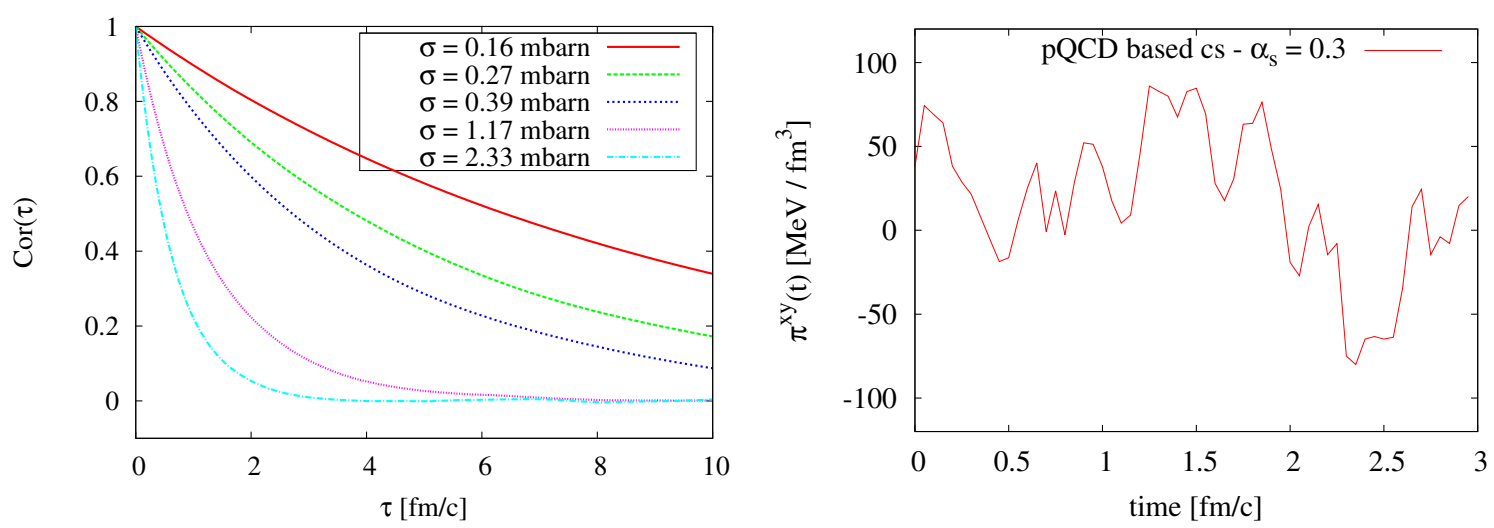

Fig. 6. Left panel: The correlation function for different isotropic cross section is shown. The relaxation time $\tau$ is linear dependent on the cross section $\sigma$.Right panel: A snapshot of a fluctuating shear stress tensor, which is used to calculate (in this case for pQCD interactions) the correlation function.

the system size, i.e. $\lambda_{\mathrm{mfp}}<<L$. After a sufficient long time scale a stationary velocity gradient $v_{\mathrm{z}}(x)$ is established.

In the non-relativistic limit the velocity gradient is linear, $d v_{\mathrm{z}}(x) / d x=$ const. Within the Navier-Stokes Ansatz the shear stress tensor in $x$-z-direction is proportional to the gradient of the velocity:

$$
\pi^{x z}=-\eta \frac{\partial v_{z}(x)}{\partial x}
$$

In the true relativistic limit the velocity gradient is not linear, but has the shape of a hyperbolic tangent

$$
v(x)=\tanh (\theta x)
$$

where the rapidity $y_{z}(x)=\theta x$ is the argument of the hyperbolic tangent and $\theta=d y_{\mathrm{z}} / d x$.

We employ BAMPS to extract the velocity profile with $v_{\text {wall }}=0.964$. We use a massless Boltzmann gas, where the interactions between particles are realized only via binary collisions and isotropic cross sections. The results are shown in Fig.5. On the left panel we show the velocity profile, which has the expected shape of a hyperbolic tangent. The results are compared to a linear fit with $\tanh (0.4 x)$ according to the expected rapidity profile shown on the right panel of Fig.5. For small velocities the system achieves the correct non-relativistic limit, where the velocity gradient is linear.

Extracting the gradient of the rapidity profile $\theta$ allows us to calculate the shear viscosity coefficient using the common relativistic relation for the shear stress tensor

$$
\pi^{x z}=-\eta \gamma \theta
$$

where $\pi^{x z}$ for a kinetic cascade can be extracted numerically (right hand side) from BAMPS

$$
\pi^{x z}(\mathbf{r}, t)=\int d^{4} p \frac{p^{1} p^{3}}{p^{0}} f(\mathbf{p}, \mathbf{r}) \cong \sum_{j=1}^{N} \frac{p_{j}^{1} p_{j}^{3}}{p_{j}^{0}} \delta\left(\mathbf{r}_{\mathbf{j}}-\mathbf{r}\right)
$$

To verify the accuracy of this method the results were compared to the analytical expression [26] for binary collisions and isotropic cross sections $\eta=1.2654 \cdot T / \sigma$, where $T$ is the temperature and $\sigma$ is the implemented cross section. We found an excellent agreement.

The use of Green - Kubo relations is another possibility to extract the shear viscosity from numerical simulations. Green - Kubo relations connect a linear transport coefficient with the integrated correlation function of the underlying flow connected with the transport coefficient. In 
EPJ Web of Conferences

\begin{tabular}{|c||c|c|c|c|}
\hline collision type & $(\eta / s)$ shear flow & $(\eta / s)$ Green - Kubo & $(\eta / s)$ Xu et al. [15] & $(\eta / s)$ El et al. [16] \\
\hline $2 \leftrightarrow 2$ & - & $1.035 \pm 0.015$ & 1.03 & 1.4 \\
\hline $2 \leftrightarrow 2$ and $2 \leftrightarrow 3$ & $0.12 \pm 0.07$ & $0.13 \pm 0.02$ & 0.13 & 0.16 \\
\hline
\end{tabular}

Table 1. $\eta / s$ values extracted from BAMPS using the shear flow and Green - Kubo relation discussed in this proceeding. Full pQCD simulations with and without inelastic $2 \leftrightarrow 3$ Bremsstrahlung processes were performed with $\alpha_{s}=0.3$ and $T=0.4 \mathrm{GeV}$. The extracted values are compared to the results of previous calculations discussed in $[15,16]$.

hydrodynamics the shear stress tensor is generated by a shear gradient. Using the Green - Kubo framework the shear viscosity $\eta$ is found to be linear with the correlation function of the equilibrium shear tensor. The presence of fluctuations is attested to every equilibrated system and Onsager's regression hypothesis [27] tells that fluctuations are driven back to equilibrium by the same transport parameter as small non-equilibrium deviations, which motivates the Green - Kubo relations. The type of interaction and its property, for example the collision rate, directly influence the emergence and decline of fluctuations. Therefore these effects also influence the shape of the correlation function which is the link to the shear viscosity. With a semianalytic discussion of the correlation function the shear viscosity of the system can be reduced to be only dependent on the mediums relaxation time of its auto correlation function. As a consequence, this relaxation time includes all interaction and medium effects.

In relativistic notation the Green - Kubo relation for the shear viscosity has the following form [28]:

$$
\begin{aligned}
\eta & =\lim _{T \rightarrow \infty} \frac{1}{2 T} \cdot \frac{V}{10 \cdot k_{B} T} \int_{-\infty}^{\infty} d \tau \int_{V} d \mathbf{r} \int_{V} d \mathbf{x} \\
& \times \int_{-T}^{T} d t\left\langle\pi^{x z}(\mathbf{x}, t) \cdot \pi^{x z}(\mathbf{x}+\mathbf{r}, t+\tau)\right\rangle,
\end{aligned}
$$

where $\pi^{x z}(\mathbf{r}, t)$ is defined above

The expression for the shear viscosity can be brought in a more convenient form when the correlation function of shear tensor is known. BAMPS solves the Boltzmann equation for an ultra relativistic gluon gas with stochastically interpreted cross sections. The observed correlation function follows an exponential decay $\operatorname{Cor}(\tau)=C_{0} \cdot \exp -t / \tau$. The zero time correlation $C_{0}$ can be derived analytically by the variance of the equilibrium Boltzmann distribution, the equilibrium gluon density and its projection on the xyplane:

$$
\operatorname{Cor}(\tau)=\int_{V} \operatorname{Cor}(\mathbf{r}, \tau=0) d \mathbf{r}=\left(\frac{4}{\pi}\right) T^{5} \cdot \frac{1}{V} \cdot \exp -t / \tau
$$

With the exponential ansatz and the zero time correlation, the Green - Kubo relation can be integrated analytically. To calculate the shear viscosity to entropy density, the equilibrium density for an ultra relativistic gas is used: $s=$ $4 n$. This will give the simplified Green - Kubo relation for BAMPS, which depends only on $\tau$ :

$$
\frac{\eta}{S}=\left(\frac{\pi}{20}\right) \cdot T \cdot \tau
$$

$\tau$ includes all interaction effects of the medium, see Fig. 6 for a correlation functions with isotropic and constant cross sections. Again, this method is tested for binary and isotropic cross sections and comparison gives an excellent agreement to the known results.

Finally, in Table 1 we now summarize the results extracted from full pQCD simulations performed with the BAMPS using both introduced methods. We use a coupling constant $\alpha_{s}=0.3$ and $T=0.4 \mathrm{GeV}$ and show the results with and without inelastic pQCD Bremsstrahlung. In addition the results are compared to previous calculations, based on Navier Stokes [15] and Grads method [16]. The results presented in [16] are extracted from full dynamical simulations, therefore loss of chemical equilibrium and the size of equilibrium deviations might have an effect on the obtained values. In contrast, the methods discussed in these proceedings employ a static setup. We observe an overall good agreement. For $\alpha_{s}=0.3$ the shear viscosity to entropy density ratio is around $\eta / s=0.13$ as raised in the introduction and in [15]; this low value is mainly due to the implementation of inelastic $2 \leftrightarrow 3$ Bremsstrahlung processes [15], which are thus of crucial relevance to understand the perfect liquid behavior of the system.

A future challenge will be to extract the shear viscosity $\eta$ for much small coupling constant $\alpha_{s}$ and to check [29] for the expected $\eta \sim \alpha_{s}^{2} \log \left(1 / \alpha_{s}\right)$ behavior [30].

\section{References}

1. S.S. Adler et al. (PHENIX), Phys. Rev. Lett. 91, 182301 (2003), nucl-ex/0305013

2. J. Adams et al. (STAR), Phys. Rev. Lett. 92, 052302 (2004), nucl-ex/0306007

3. B.B. Back et al. (PHOBOS), Phys. Rev. C72, 051901 (2005), nucl-ex/0407012

4. P. Kovtun, D.T. Son, A.O. Starinets, Phys. Rev. Lett. 94, 111601 (2005), hep-th/0405231

5. J. Adams et al. (STAR), Phys. Rev. Lett. 91, 172302 (2003), nucl-ex/0305015

6. C. Adler et al. (STAR), Phys. Rev. Lett. 89, 202301 (2002), nucl-ex/0206011

7. K. Adcox et al. (PHENIX), Phys. Rev. Lett. 88, 022301 (2002), nucl-ex/0109003

8. F. Wang (STAR), J. Phys. G30, S1299 (2004), nucl-ex/0404010

9. H. Stoecker, Nucl. Phys. A750, 121 (2005), nucl-th/0406018

10. Z. Xu, C. Greiner, Phys. Rev. C71, 064901 (2005), hep-ph/0406278 
11. A. El, Z. Xu, C. Greiner, Nucl. Phys. A806, 287 (2008), 0712. 3734

12. O. Fochler, Z. Xu, C. Greiner, Phys. Rev. Lett. 102, 202301 (2009), 0806.1169

13. Z. Xu, C. Greiner, H. Stocker, Phys. Rev. Lett. 101, 082302 (2008), 0711.0961

14. Z. Xu, C. Greiner, Phys. Rev. C79, 014904 (2009), 0811.2940

15. Z. Xu, C. Greiner, Phys. Rev. Lett. 100, 172301 (2008), 0710.5719

16. A. El, A. Muronga, Z. Xu, C. Greiner, Phys. Rev. C79, 044914 (2009), 0812.2762

17. S. Wicks, W. Horowitz, M. Djordjevic, M. Gyulassy, Nucl. Phys. A784, 426 (2007), nucl-th/0512076

18. I. Bouras et al., Phys. Rev. Lett. 103, 032301 (2009), Q902. 1927

19. I. Bouras et al., Nucl. Phys. A830, 741c (2009), 0907.4519

20. I. Bouras et al., Phys. Rev. C82, 024910 (2010), 1006.0387

21. A. Adare et al. (PHENIX), Phys. Rev. Lett. 101, 232301 (2008), 0801.4020

22. O. Fochler, Z. Xu, C. Greiner (2010), 1003.4380

23. B.I. Abelev et al. (STAR), Phys. Rev. C77, 054901 (2008), 0801.3466

24. W. Scheid, H. Müller, W. Greiner, Phys. Rev. Lett. 32(13), 741 (1974)

25. I. Bouras et al., publication in preparation (2011)

26. S.R. de Groot et al., Relativistic Kinetic Theory: Principles and Applications (North Holland, Amsterdam, 1980)

27. L. Onsager, Phys. Rev. 37(4), 405 (1931)

28. D.N. Zubarev, V. Morozov, G. Ropke, Statistical Mechanics of Nonequilibrium Processes: Relaxation and Hydrodynamic Processes (Akademie Verlag, Berlin, 1997)

29. F. Reining, C. Wesp, publications in preparation (2011)

30. G. Baym, H. Monien, C.J. Pethick, D.G. Ravenhall, Phys. Rev. Lett. 64(16), 1867 (1990) 